\title{
Age-dependent insulin resistance in male mice with null deletion of the carcinoembryonic antigen-related cell adhesion molecule 2 gene
}

\author{
Simona S. Ghanem ${ }^{1}$ - Harrison T. Muturi ${ }^{2}$ Anthony M. DeAngelis ${ }^{1}$. \\ Jiang $\mathrm{Hu}^{3} \cdot$ Rohit N. Kulkarni ${ }^{3}$. Garrett Heinrich ${ }^{2,4}$ - Sonia M. Najjar ${ }^{1,2,4}$
}

Received: 11 January 2017 / Accepted: 21 April 2017 /Published online: 31 May 2017

(C) Springer-Verlag Berlin Heidelberg 2017

\begin{abstract}
Aims/hypothesis $\mathrm{Cc}^{-/-}$mice lacking the gene encoding the carcinoembryonic-antigen-related cell adhesion molecule 2 ( $C c 2$ [also known as Ceacam2]) exhibit hyperphagia that leads to obesity and insulin resistance. This starts at 2 months of age in female mice. Male mutants maintain normal body weight and insulin sensitivity until the last age previously examined (7-8 months), owing to increased sympathetic tone to white adipose tissue and energy expenditure. The current study investigates whether insulin resistance develops in mutant male mice at a later age and whether this is accompanied by changes in insulin homeostasis.

Methods Insulin response was assessed by insulin and glucose tolerance tests. Energy balance was analysed by indirect calorimetry.
\end{abstract}

Electronic supplementary material The online version of this article (doi:10.1007/s00125-017-4307-2) contains peer-reviewed but unedited supplementary material, which is available to authorised users.

Sonia M. Najjar

najjar@ohio.edu

1 Center for Diabetes and Endocrine Research, College of Medicine and Life Sciences, University of Toledo, Toledo, OH, USA

2 Department of Biomedical Sciences, Heritage College of Osteopathic Medicine, Ohio University, Athens, OH, USA

3 Section on Islet Cell and Regenerative Biology, Joslin Diabetes Center and Department of Medicine, Brigham and Women's Hospital, Harvard Medical School, Harvard Stem Cell Institute, Boston, MA, USA

4 Diabetes Institute, Heritage College of Osteopathic Medicine, Ohio University, Athens, OH 45701-2979, USA
Results Male $\mathrm{Cc}_{\mathrm{C}}{ }^{-/}$mice developed overt metabolic abnormalities at about 9 months of age. These include elevated global fat mass, hyperinsulinaemia and insulin resistance (as determined by glucose and insulin intolerance, fed hyperglycaemia and decreased insulin signalling pathways). Pair-feeding experiments showed that insulin resistance resulted from hyperphagia. Indirect calorimetry demonstrated that older mutant male mice had compromised energy expenditure. Despite increased insulin secretion caused by $C c 2$ deletion, chronic hyperinsulinaemia did not develop in mutant male mice until about 9 months of age, at which point insulin clearance began to decline substantially. This was probably mediated by a marked decrease in hepatic CEACAM1 expression.

Conclusions/interpretation The data demonstrate that at about 9 months of age, $C c 2^{-/-}$male mice develop a reduction in energy expenditure and energy imbalance which, combined with a progressive decrease in CEACAM1-dependent hepatic insulin clearance, causes chronic hyperinsulinaemia and sustained age-dependent insulin resistance. This represents a novel mechanistic underpinning of age-related impairment of hepatic insulin clearance.

Keywords CEACAM2 - Energy balance .

Hyperinsulinaemia $\cdot$ Hyperphagia $\cdot$ Insulin clearance $\cdot$ Insulin resistance $\cdot$ Insulin secretion
Abbreviations
$\mathrm{Cc}^{-/-} \quad$ Ceacam2 homozygous null mutant
$\mathrm{CC} 2^{+/+}$
Wild-type littermate
CEACAM Carcinoembryonic antigen-related cell adhesion molecule
GLP-1 Glucagon-like peptide 1
$\mathrm{IR}_{\beta} \quad \quad \quad \beta$ Subunit of the insulin receptor 
qRT-PCR Quantitative real-time PCR

VMH Ventromedial nucleus of the hypothalamus

$\dot{V} \mathrm{O}_{2} \quad$ Oxygen consumption

\section{Introduction}

The carcinoembryonic-antigen-related cell adhesion molecule (CEACAM)2 is a transmembrane glycoprotein that belongs to the carcinoembryonic antigen (CEA) family $[1,2]$. Its gene is distinct from that encoding CEACAM1, another member of this family of proteins that is ubiquitously expressed [3] and that regulates insulin sensitivity by promoting insulin clearance $[4,5]$, which predominantly occurs in liver and to a lesser extent in kidney [3].

CEACAM2 is expressed in kidney, spleen, testis and platelets in addition to the crypt cells and the villi lining of the intestinal segment beginning with distal jejunum [6-12]. It is also expressed in the hypothalamus, including the ventromedial hypothalamus (VMH), and other brain centres involved in feeding behaviour, including hippocampus, striatum, olfactory bulb and the globus and ventral pallidus [6, 13, 14]. Consistently, the metabolic phenotype of mice with null mutation of $C \mathrm{c} 2$ (also known as Ceacam2) gene $\left(\mathrm{Cc}^{-/-}\right)$shows that CEACAM2 is involved in the central regulation of food intake and energy expenditure, and in the sympathetic regulation of brown adipogenesis [6, 15].

Whereas CEACAM2 is detected in pancreatic cells, in particular in non-beta cells, its null deletion does not influence basal plasma levels of hormones (insulin, glucagon and somatostatin), nor does it affect islet areas or glucose-induced insulin secretion in pooled $C c 2^{-/-}$islets [12]. Instead, CEACAM2 promotes glucose-stimulated insulin secretion. This is mediated at least in part via a mechanism dependent on glucagon-like peptide-1 (GLP-1), consistent with its expression in the distal intestinal villi [12]. In addition to its role in metabolism, CEACAM2 regulates spermatid maturation [7, 16] and platelet activation and adhesion [8].

Both male and female $C c 2^{-/-}$mice display hyperphagia. Given that plasma leptin level is normal at the onset of hyperphagia in both sexes, it is more reasonable to predict that hyperphagia in $C c 2^{-/-}$mice is primarily caused by the absence of CEACAM2 from brain centres that control feeding behaviour and rewards instead of changes in leptin sensitivity [17]. This notion is supported by the induction of brain CEACAM2 production that occurs at fasting and its suppression at refeeding [6]. Studies in GLUTag entero-endocrine cells revealed that glucose, but not insulin, rapidly suppresses $C c 2$ mRNA level at refeeding [12].

Hyperphagia causes obesity and insulin resistance in female $C c 2^{-1-}$ mice at 2 months of age, as demonstrated by pair-feeding experiments [6]. In contrast, it fails to trigger insulin resistance in male mutants up to 7 months of age [12,
15], probably because of the differential hypermetabolic state in relation to activated sympathetic tone regulation of white adipose tissue (WAT) and increased spontaneous activity in male mutants [15]. $C c 2^{-/-}$male mice remain lean and insulin sensitive until 7-8 months of age, as demonstrated by hyperinsulinaemic-euglycaemic clamp analysis [12]. They also manifest elevated glucose-induced glucagon-like peptide 1 (GLP-1)-mediated insulin secretion with preserved beta cell insulin secretory function at this age, as shown by hyperglycaemic clamp analysis [12]. Thus, the current study investigated whether chronic hyperphagia eventually causes insulin resistance in older male mice, and whether this is accompanied by changes in insulin homeostasis.

\section{Methods}

Animals The generation and propagation of $\mathrm{Cc}^{-/-}$mice on a C57BL/6J genetic background (Jackson Laboratory, Bar Harbor, ME, USA) has been previously described [6]. Male mice (5-12 months of age) were housed with a $12 \mathrm{~h}$ dark/light cycle, fed standard chow (Harlan, Teklad 2016, Harlan, Haslett, MI, USA) ad libitum and kept in specific pathogenfree conditions at the Division of Laboratory Animal Resources at the University of Toledo. The Institutional Animal Care and Utilization Committee (IACUC) approved all procedures and animal experiments. For fasting, mice were transferred from corn cob bedding to Alpha-dri Bedding (Shepherd, Watertown, TN, USA).

Body composition Whole-body composition was assessed in awake mice by NMR technology (Bruker Minispec, Billerica, MA, USA) [18]. Fat and lean mass were measured as percentage of total body weight.

Metabolic variables Retro-orbital venous blood was drawn at 11:00 h from overnight-fasted mice under pentobarbital anaesthesia $(1.1 \mathrm{mg} / \mathrm{kg}$ body weight) to measure plasma insulin (80-INSMSU-E01, ALPCO, Salem, NH, USA), C-peptide (80-CPTMS-E01, ALPCO) and total GLP-1 levels (GLP1T36HK, Millipore, Temecula, CA, USA). The steady-state C-peptide/insulin molar ratio was calculated as a surrogate marker for insulin clearance [4, 18]. Whole-blood glucose measurements from tail snipping were made with a glucometer from overnight-fasted and randomly fed mice (Accu-Chek, Roche, Pleasanton, CA, USA). Plasma NEFA (NEFA-C; Wako, Richmond, VA, USA) and triacylglycerol (Pointe Scientific, Canton, MI, USA) were also measured. Tissue triacylglycerol content was assayed, as described previously [18].

Intraperitoneal insulin tolerance test Animals were fasted for $5 \mathrm{~h}$ starting at 07:00 h before being subjected to an i.p. injection of Human Insulin Novolin (Novo Nordisk NDC 
0169-1833-11, Plainsboro, NJ, USA) at $0.75 \mathrm{U} / \mathrm{kg}$ body weight. Blood glucose was measured from the tail vein at 0-180 min post insulin injection.

Glucose tolerance test At 5 days after the insulin tolerance test, animals were fasted overnight from 17:00 $\mathrm{h}$ to $08: 00 \mathrm{~h}$ before receiving either an i.p. injection $(1.5 \mathrm{~g} / \mathrm{kg}$ body weight) or an oral gavage ( $3 \mathrm{~g} / \mathrm{kg}$ body weight) of glucose (50\% [wt/vol.] dextrose solution). Blood glucose level was measured from the tail vein of awake mice at 0-120 min post glucose administration.

Glycogen assay As previously described [19], livers were removed from mice fasted and refed for $7 \mathrm{~h}$, digested $(100 \mathrm{mg})$ in $6 \%$ (wt/vol.) perchloric acid, and incubated in $1 \mathrm{mg} / \mathrm{ml}$ amyloglucosidase for $2 \mathrm{~h}$ at $42^{\circ} \mathrm{C}$. Glycogen assay reagent (Sigma, Saint Louis, MO, USA) was added and samples were incubated at room temperature for $15 \mathrm{~min}$, analysed at $\mathrm{A}_{340} \mathrm{~nm}$ and readings were converted to $\mu \mathrm{g} / \mathrm{mg}$ wet tissue weight.

Western blot analysis Proteins were separated by $7 \%$ SDS-PAGE and immunoprobed with: polyclonal antibodies $(1: 1000)$ against phospho-insulin receptor $\left(\mathrm{pIR}_{\beta}\right)$ (phosphoY1361) and $\mathrm{IR}_{\beta}$ (C18C4) (Abcam, Cambridge, MA, USA), phospho-Ser473Akt and Akt (Cell Signaling, Danvers, MA, USA); and custom-made polyclonal antibodies raised in rabbit against mouse CEACAM1 ( $\alpha$-mCC1 Ab3759) and phosphoCEACAM1 ( $\alpha$-pCC1) (Bethyl Laboratories, Montgomery, TX, USA), as previously titrated and optimised [18]. Blots were incubated with horseradish peroxidase-conjugated sheep anti-mouse or donkey anti-rabbit IgG antibody (GE Healthcare Life Sciences, Amersham, Sunnyvale, CA, USA), followed by enhanced chemiluminescence.

Pair feeding The average daily food intake was assessed in individually caged 9 month old mice over a 5 day period before mutants were subjected to a pair-feeding regimen: $3.2 \mathrm{~g}$ food/day ( $0.5 \mathrm{~g}$ less than the $C c 2^{-/-}$mice fed ad libitum) for the first week, followed by $3.7 \mathrm{~g}$ vs $5.5 \mathrm{~g}$ of food/day to the ad libitum-fed group in the second week.

Insulin secretion Overnight-fasted mice were anaesthetised with pentobarbital $(1.1 \mathrm{mg} / \mathrm{kg}$ body weight) before receiving an i.p. glucose injection ( $3 \mathrm{~g} / \mathrm{kg}$ body weight). Retro-orbital blood was drawn at $0-120 \mathrm{~min}$ post glucose injection to measure insulin levels.

Immunofluorescence analysis Mice were anaesthetised with pentobarbital ( $1.1 \mathrm{mg} / \mathrm{kg}$ body weight), and pancreases were dissected, weighed, fixed in Z-fixative, sectioned and immunostained with anti-human insulin (Linco Research, Billerica, MA, USA) and anti-mouse glucagon monoclonal antibodies (Sigma), followed by aminomethylcoumarin (AMCA)- conjugated donkey and Texas Red-conjugated donkey antibody (Jackson Immunoresearch, West Grove, PA, USA), respectively [20]. Alpha and beta cell areas were calculated by morphometric analysis using ImageJ software (http://rsb.info. nih.gov/ij/) [21, 22], and the glucagon/insulin-stained area was divided by total pancreas area.

Indirect calorimetry Indirect calorimetry was measured in awake mice ( $n=5 /$ genotype), as described previously [18]. Mice were individually caged (CLAMS system, Columbus Instrument, Columbus, OH, USA) for 3 days, after being acclimatised for 2 days, and had free access to food and water. Spontaneous locomotor activity was measured with an optical beam device quantifying horizontal and vertical movement $\left(x\right.$ -,$y$ - and $z$-axes). Oxygen consumption $\left(\dot{V} \mathrm{O}_{2}\right)$ and heat production (energy expenditure) were measured every $20 \mathrm{~min}$ and data were normalised per fat-free lean mass.

Quantitative real-time-PCR Total RNA was isolated with PerfectPure RNA Tissue Kit (Fisher Scientific, Waltham, MA, USA). cDNA was synthesised by iScript cDNA Synthesis Kit (Bio-Rad, Hercules, CA, USA), using $1 \mu \mathrm{g}$ total RNA and oligodT primers (electronic supplementary material [ESM] Table 1). cDNA was evaluated with quantitative realtime PCR (qRT-PCR) (StepOne Plus, Applied Biosystems, Foster City, CA, USA), and mRNA was normalised to Gapdh, as previously described [18].

Statistical analysis Data were analysed using one-factor ANOVA analysis with Bonferroni correction or Student's $t$ test and presented as means \pm SEM. Values of $p<0.05$ were statistically significant.

\section{Results}

Age-dependent changes in body composition of $\mathrm{Cc}^{-/-}$male mice Consistent with previous reports [6, 12, 15], 5 month old $C c 2^{-/-}$male mice had body weight comparable with their wild-type littermate $\left(C c 2^{+/+}\right)$counterparts (Table 1$)$. This was accompanied by elevated lean mass, with a reciprocal reduction in fat mass, as assessed by NMR analysis (Table 1). Starting at 9 months of age, however, male mutants began to gain more body weight (Table 1), with a significant increase in fat mass and a reciprocal reduction in lean mass in comparison with age-matched wild types (Table 1).

Age-dependent changes in insulin sensitivity and glucose tolerance of $C \boldsymbol{c} 2^{-1-}$ male mice As expected from hyperinsulinaemic-euglycaemic clamp analysis [12, 15], 5 month old $\mathrm{Cc}^{-/-}$male mice exhibited improved glucose and insulin tolerance (Fig. 1a). However, starting at 9 months, 
Table 1 Plasma and tissue biochemistry in male mice at different ages

\begin{tabular}{|c|c|c|c|c|c|c|}
\hline \multirow[t]{2}{*}{ Characteristic } & \multicolumn{2}{|l|}{5 months } & \multicolumn{2}{|l|}{9 months } & \multicolumn{2}{|l|}{12 months } \\
\hline & $C c 2^{+/+}$ & $\mathrm{Cc}^{-/-}$ & $C c 2^{+/+}$ & $C c 2^{-/-}$ & $C c 2^{+/+}$ & $C c 2^{-/-}$ \\
\hline Body weight (g) & $28.7 \pm 0.3$ & $29.9 \pm 0.4$ & $30.9 \pm 0.2$ & $40.6 \pm 2.8^{*}$ & $33.7 \pm 0.7$ & $44.2 \pm 5.0^{*}$ \\
\hline$\%$ Fat mass & $9.2 \pm 0.8$ & $0.9 \pm 0.1^{*}$ & $11.5 \pm 0.6$ & $18.3 \pm 2.1^{*}$ & $12.4 \pm 0.1$ & $22.1 \pm 0.2^{*}$ \\
\hline$\%$ Lean mass & $64.7 \pm 0.6$ & $73.2 \pm 0.7^{*}$ & $63.7 \pm 0.5$ & $58.3 \pm 1.8^{*}$ & $62.2 \pm 0.1$ & $56.0 \pm 0.2^{*}$ \\
\hline Fasting glucose $(\mathrm{mmol} / \mathrm{l})$ & $4.8 \pm 0.1$ & $4.4 \pm 0.3$ & $4.6 \pm 0.1$ & $5.1 \pm 0.3$ & $4.7 \pm 0.1$ & $5.1 \pm 0.4$ \\
\hline Fed glucose $(\mathrm{mmol} / \mathrm{l})$ & $9.3 \pm 0.2$ & $8.6 \pm 0.2$ & $8.9 \pm 0.3$ & $10.1 \pm 0.2^{*}$ & $9.1 \pm 0.3$ & $10.3 \pm 0.4^{*}$ \\
\hline Plasma NEFA (mmol/l) & $0.4 \pm 0.0$ & $0.2 \pm 0.0^{*}$ & NA & NA & $0.4 \pm 0.0$ & $0.5 \pm 0.0^{* \dagger}$ \\
\hline Plasma TG (mmol/l) & $0.51 \pm 0.01$ & $0.35 \pm 0.01^{*}$ & NA & NA & $0.54 \pm 0.01$ & $0.76 \pm 0.01^{* \dagger}$ \\
\hline $\begin{array}{l}\text { Skeletal muscle } \\
\text { TG }(\mu \mathrm{g} / \mathrm{mg})\end{array}$ & $4.5 \pm 0.1$ & $2.8 \pm 0.1^{*}$ & NA & NA & $4.7 \pm 0.1$ & $6.6 \pm 0.1^{* \dagger}$ \\
\hline Hepatic TG $(\mu \mathrm{g} / \mathrm{mg})$ & $56.4 \pm 0.6$ & $55.8 \pm 0.7$ & NA & NA & $57.5 \pm 0.8$ & $55.6 \pm 0.9$ \\
\hline Plasma GLP-1 (pmol/1) & $1.2 \pm 0.1$ & $4.2 \pm 0.8^{*}$ & $1.3 \pm 0.2$ & $4.0 \pm 0.9^{*}$ & $0.9 \pm 0.2$ & $4.6 \pm 0.7^{*}$ \\
\hline
\end{tabular}

Male mice ( $n=5-6 /$ genotype) were fasted overnight before retro-venous blood was drawn

Values are expressed as means \pm SEM

${ }^{*} p<0.05$ vs $C c 2^{+/+}$of the same age group; ${ }^{\dagger} p<0.05$ vs 5 month old $C c 2^{-/-}$mice

NA, not applicable; TG, triacylglycerol

they began to show intolerance to exogenous insulin (Fig. 1d) and glucose (administered both i. p. [Fig. 1c] and orally ESM Fig. 1]). They also started exhibiting elevated fed, but not fasting, glucose levels in comparison with $\mathrm{Cc}^{+/+}$mice, with a rise in plasma NEFA (Table 1).

Consistent with the emergence of insulin resistance at this age, insulin excursion of approximately two to fourfold with $7 \mathrm{~h}$ of refeeding following an overnight fast induced phosphorylation of $\mathrm{IR}_{\beta}$ and Akt in the liver (Fig. 2a) and skeletal muscle (Fig. 2b) of 5 month old but not 9 month old $C c 2^{-/-}$ mice, as demonstrated by sequential immunoblotting with antibodies against phosphorylated antibodies followed by reimmunoblotting with antibodies against these proteins. Similarly, CEACAM1 phosphorylation in response to insulin was blunted in mutant mice livers, starting at 9 months (Fig. 2a).

\section{Hyperphagia drives insulin resistance in older $C c 2^{-/-}$male} mice $C c 2^{-1-}$ male mice exhibited a significant increase in daily food consumption at all ages (Fig. 2c; only 9 month old mice are shown). This contributes to their higher body weight than $C c 2^{+/+}$mice fed ad libitum (Fig. 2d). Subjecting them to a pair-feeding regimen for 2 weeks to restrict their food intake and reduce their body mass to a level comparable to ad libitum-fed $\mathrm{Cc}^{+/+}$mice (Fig. $2 \mathrm{~d}$ ) restored their tolerance to exogenous insulin (Fig. 2e). This demonstrates that insulin resistance in older $C c 2^{-/-}$males is primarily caused by hyperphagia.

Decreased energy expenditure in older $C c 2^{-/-}$male mice Indirect calorimetry analysis (Fig. 3) revealed that $C c 2^{-/-}$ mice had higher energy expenditure (not shown) and
$\dot{V} \mathrm{O}_{2}$ consumption than age-matched $\mathrm{Cc}^{+/+}$males in both light and dark cycles (shaded) at 5 months of age (Fig. 3a), as previously shown [15]. Spontaneous physical activity was also elevated in the 5 month old mutants (Fig. 3b). However, starting at about 8 months, mutant mice began to exhibit a decrease in energy expenditure (not shown) and $\dot{V} \mathrm{O}_{2}$ consumption (Fig. 3c, e) without a significant change in locomotor activity (Fig. 3d) until about 12 months of age, at which point their physical activity in the dark cycle declined, to become lower than in wild-type mice (Fig. 3f).

Consistent with the skeletal muscle being a major site of energy expenditure, mRNA levels of genes involved in fatty acid transport (Cd36, Fatpl [also known as Slc27a1]) and $\beta$-oxidation (Ppara [also known as Ppara], Cpt1b, Cox2, Ucp3) were higher in the 5 month old mutants than wild types, but were lower at 12 months (Table 2). Consistently, the skeletal muscle triacylglycerol content was higher in 12 month old mutants relative to wild types (Table 1). This could be, in part, related to a higher sympathetic nervous outflow to skeletal muscle at 5 months of age that became lower at 12 months, as suggested by the mRNA levels of the surrogate marker, the adrenergic- $\beta$-receptor 2 (Adrb2) (Table 2) [23, 24].

We have shown that 5 month old $C c 2^{-/-}$male mice exhibit an increase in sympathetic tone to WAT [15]. Consistently, mRNA levels of $U c p 1$, the adrenergic $\beta$ receptors 2 and 3 (Adrb2 and Adrb3) and type 2 deiodinase (Dio2), a key gene in brown adipose tissue [25], are elevated in gonadal WAT from 5 month old, but not 12 month old, mutants (Table 2). This agrees with insulin sensitivity, the overall lean phenotype and the higher basal metabolic rate in 5 month old, but not 12 month old, $C c 2^{-/-}$male mice. 

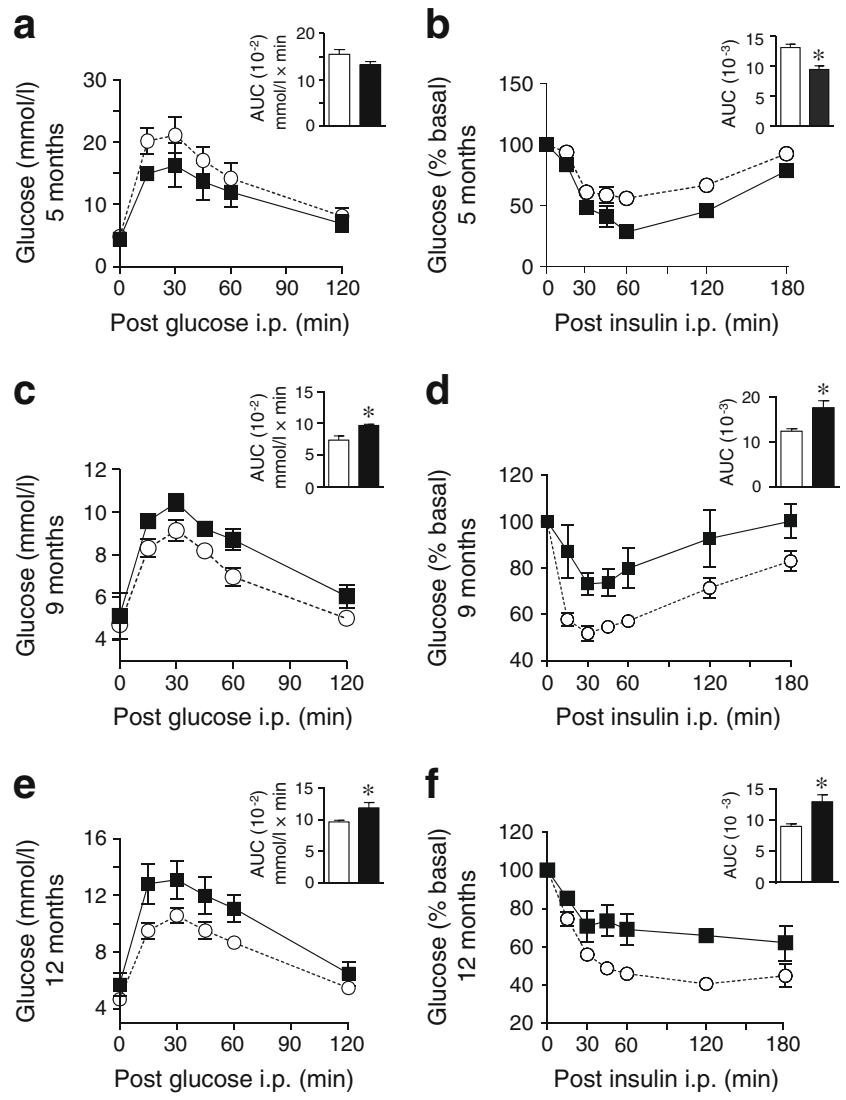

Fig. 1 Intraperitoneal glucose and insulin tolerance tests. Male mice (5-12 months of age, $n \geq 5$ for each genotype and age group) were subjected to i.p. injections of glucose ( $1.5 \mathrm{~g} / \mathrm{kg}$ body weight) (a, c, e) or insulin $(0.75 \mathrm{U} / \mathrm{kg}$ body weight $)(\mathbf{b}, \mathbf{d}, \mathbf{f})$ to assess blood glucose levels at 0-120 and 0-180 min post injection, respectively. AUC for the glucose $(\mathrm{mmol} / \mathrm{l} \times \mathrm{min})$ and insulin (arbitrary units) was measured and represented in bar graphs. Values are expressed as means \pm SEM. ${ }^{*} p<0.05$ vs $C c 2^{+/+}$mice. Black squares and bars, $\mathrm{Cc}^{-/-}$; white circles and bars, $\mathrm{Cc} 2^{+/+}$

Sustained enhanced insulin secretion in aged $C c 2^{-/-}$male mice $C c 2^{-/-}$male mice manifested higher basal and oral glucose-induced GLP-1 levels than age-matched wild-type mice at 5 months of age [12]. As Table 1 shows, plasma GLP-1 remained elevated in mutant mice up to 12 months of age. Throughout all age groups, mutant mice had higher acute insulin release in response to i.p. injection of glucose relative to wild-type $C \mathrm{C}^{+/+}$mice (Fig. 4a). The slight rise in insulin excursion at 120 min post injection beginning at 9 months (Fig. 4b) is consistent with the development of insulin resistance at this age. Of note, the increase in insulin secretory function occurred without any significant change in either alpha or beta islet cell area relative to total pancreas in $C c 2^{-/}$mice, even at 12 months of age (Fig. $4 \mathrm{~d}-\mathrm{g}$ ).

More robust age-dependent progressive decrease in hepatic CEACAM1 in older $\boldsymbol{C} \boldsymbol{c} 2^{-/-}$male mice Despite the rise in insulin secretion, plasma insulin level was normal in 5 month old mice, consistent with insulin sensitivity at this age [15].
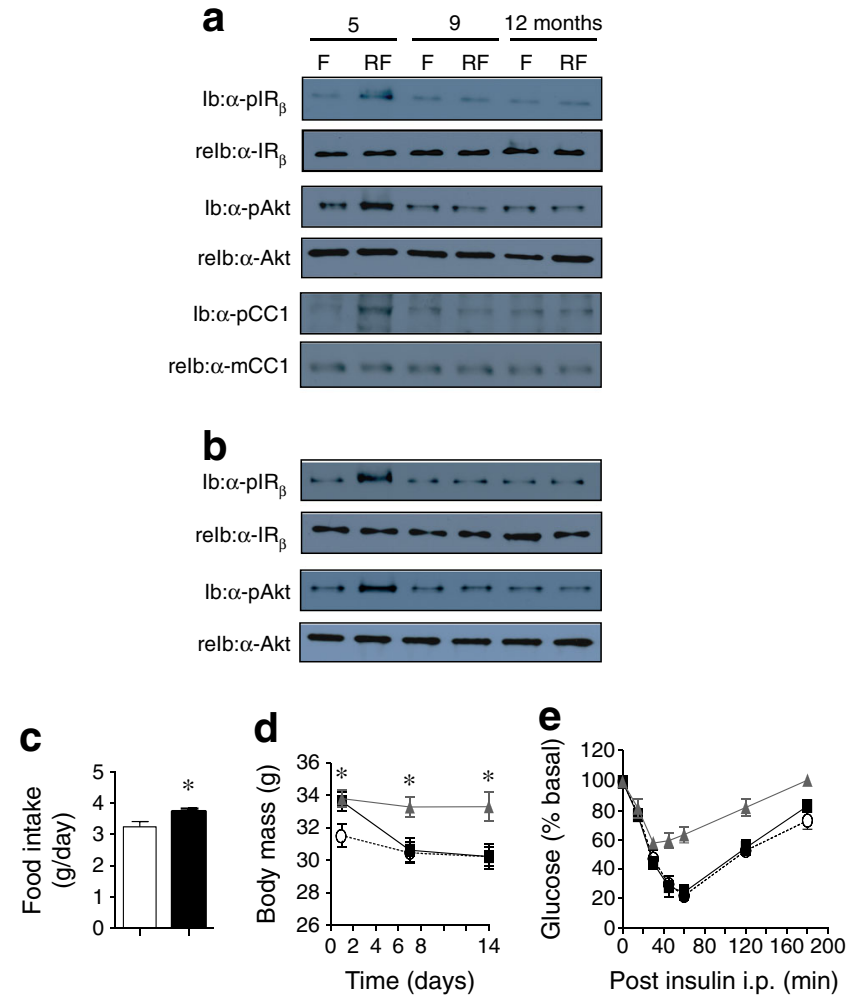

Fig. 2 Insulin signalling and pair-feeding experiments. (a, b) Phosphorylation (and activation) of $\mathrm{IR}_{\beta}$, Akt and CEACAM1 (CC1) in response to insulin excursion during refeeding for $7 \mathrm{~h}$ following an overnight fast was assessed by immunoblotting proteins using antibodies to phosphorylated proteins $(\alpha$-pIR $\beta, \alpha$-pAkt and $\alpha$-pCC1) in lysates from liver (a) and skeletal muscle (b) of $C c 2^{--}$mutants at 5,9 and 12 months of age. Re-immunoblotting with $\mathrm{IR}_{\beta}$, Akt and CEACAM1 antibodies was performed to normalise the amount of loaded proteins. Each set is a representative of three independent experiments performed on different mice. (c) Daily food consumption over 5 consecutive days demonstrated hyperphagia in 9-month-old $C c 2^{-/-}$mice (black bar) vs $C c 2^{+/+}$mice (white bar) ( $n=5 /$ genotype). (d, e) Pair-feeding experiments were performed on 9-month-old mice. (d) While some $C c 2^{-/-}$mutants were fed ad libitum, some were subjected to a pair-feeding regimen for 2 weeks to decrease their body weight to the level of ad libitum-fed $C c 2^{+/+}$mice ( $n=5$ for each genotype and feeding group). (e) At the end of the feeding period, insulin tolerance was determined. Values are expressed as means $\pm \mathrm{SEM}$ at each time point. Black squares, pair-fed $\mathrm{Cc}^{-/-}$mutants; grey triangles, $C c 2^{-/}$mutants fed ad libitum; white circles, $C c 2^{+/+}$mice fed ad libitum. ${ }^{*} p<0.05$ vs ad libitum-fed $C c 2^{+/+}$. F, fasted; Ib, immunoblot; reIb, re-immunoblot; RF, refed

This was probably a consequence of the approximately twofold increase in hepatic insulin clearance, as assessed by steady-state C-peptide/insulin molar ratio (Fig. 5c). At 9 months, chronic hyperinsulinaemia began to develop in male mutant mice, in parallel to the gradual reduction in insulin clearance (Fig. 5c). Mechanistically, the age-related decline in hepatic insulin clearance is probably due to reduced hepatic content of CEACAM1 (Fig. 5d) which, on its phosphorylation by the receptor, promotes receptor-mediated insulin uptake by the hepatocyte to be targeted for degradation [4, 5]. Mice with liver-specific inactivation or with null deletion of $C c 1$ gene 
a

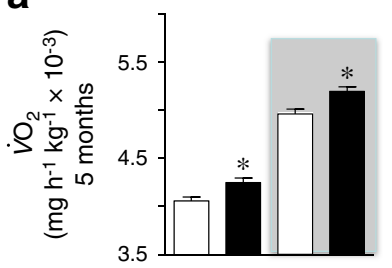

C

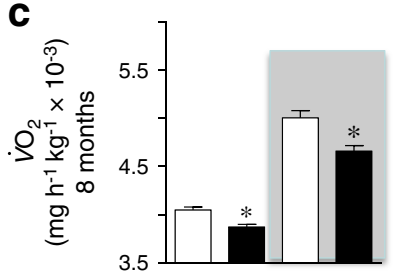

e

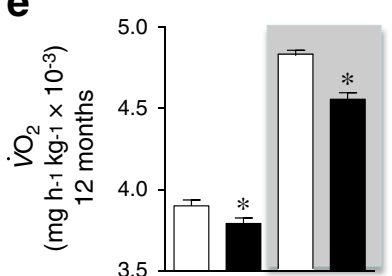

b

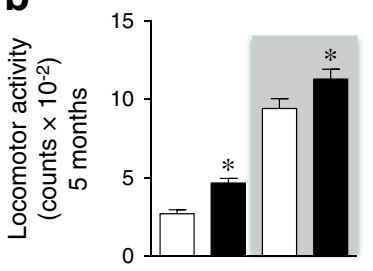

d

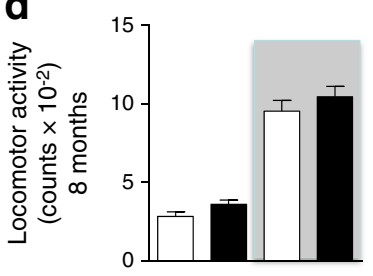

f

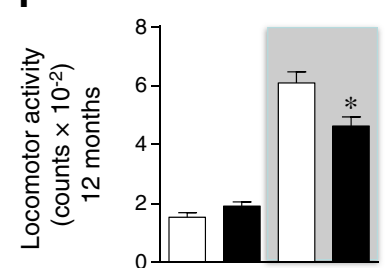

Fig. 3 Indirect calorimetry analysis. $C c 2^{+/+}$and $C c 2^{-/-}$mice ( $n=5 /$ genotype $)$ were individually caged, given free access to food and subjected to indirect calorimetry to analyse $\dot{V} \mathrm{O}_{2}(\mathbf{a}, \mathbf{c}, \mathbf{e})$, and spontaneous locomotor activity (b, d, f) over the $24 \mathrm{~h}$ period (starting at 06:00 h). Graphs show average hourly data over each $12 \mathrm{~h}$ period of the light (06:00-18:00 h) and dark (18:00-06:00 h) (shaded) cycles during the last 3 consecutive days. Values are expressed as means \pm SEM. Black bars, $C c 2^{-/-}$; white bars, $C c 2^{+/+} ;{ }^{*} p<0.05$ vs $C c 2^{+/+}$mice

(also known as Ceacam1) manifest chronic hyperinsulinaemia resulting from impaired insulin clearance $[4,5]$. Of note, hepatic $C c 1$ mRNA level appears to decline progressively with age in both genotypes, reaching $25-50 \%$ of the original level at 12 months of age (Fig. 5d).

The reduction in hepatic CEACAM1 level in $\mathrm{Cc}^{-/-}$liver at an older age is probably due to blunted insulin-induced transcription of $C c l$ under conditions of insulin resistance (Fig. 5d) [26]. It could also be due to the rise in hepatic Ppara mRNA (encoding peroxisome proliferator-activated receptor [PPAR] $\alpha$; Table 2) and its activation by elevated levels of plasma NEFA (Table 1) that are transported into the hepatocyte via cluster determinant 36 (CD36; Table 2) [27], as suggested by the increase in mRNA level of PPAR $\alpha$ 's transcriptional targets (Cpt1 and $C d 36)$ in 12 month old mutant mice (Table 2). The decrease in hepatic CEACAM1 level (and phosphorylation [Fig. 2a]) and increase in the expression of genes involved in fatty acid $\beta$-oxidation (Ppar $\alpha, C p t 1)$ is consistent with restricted lipid accumulation in the liver of older mutants (Table 1) [27].

Insulin suppresses hepatic glucose production by inhibiting gluconeogenesis and stimulating net hepatic glucose uptake and subsequent glycogen synthesis [28]. A higher fasting

Table 2 qRT-PCR analysis of mRNA levels of genes involved in fat metabolism, brown adipogenesis and gluconeogenesis

\begin{tabular}{|c|c|c|c|c|}
\hline \multirow[b]{2}{*}{ mRNA } & \multicolumn{2}{|l|}{5 months } & \multicolumn{2}{|l|}{12 months } \\
\hline & $C c 2^{+/+}$ & $\mathrm{Cc}^{-/-}$ & $C c 2^{+/+}$ & $\mathrm{Cc}^{-/-}$ \\
\hline \multicolumn{5}{|c|}{ Skeletal muscle } \\
\hline$C d 36$ & $0.52 \pm 0.04$ & $1.16 \pm 0.05^{*}$ & $0.42 \pm 0.05$ & $0.17 \pm 0.02^{* \dagger}$ \\
\hline Fatp1 & $0.72 \pm 0.05$ & $1.79 \pm 0.10^{*}$ & $0.62 \pm 0.04$ & $0.30 \pm 0.04^{* \dagger}$ \\
\hline Ppara & $0.55 \pm 0.03$ & $1.40 \pm 0.08^{*}$ & $0.48 \pm 0.05$ & $0.21 \pm 0.03^{*+}$ \\
\hline Cpt1b & $0.76 \pm 0.01$ & $1.07 \pm 0.03^{*}$ & $0.64 \pm 0.05$ & $0.36 \pm 0.02^{* \dagger}$ \\
\hline $\operatorname{Cox} 2$ & $0.62 \pm 0.02$ & $1.23 \pm 0.02^{*}$ & $0.51 \pm 0.01$ & $0.31 \pm 0.01^{* \dagger}$ \\
\hline Ucp3 & $0.58 \pm 0.03$ & $1.30 \pm 0.02^{*}$ & $0.54 \pm 0.02$ & $0.28 \pm 0.01^{* \dagger}$ \\
\hline$P d k 4$ & $0.56 \pm 0.01$ & $1.03 \pm 0.05^{*}$ & $0.60 \pm 0.02$ & $0.38 \pm 0.02^{* \dagger}$ \\
\hline Adrb2 & $1.27 \pm 0.04$ & $2.73 \pm 0.14^{*}$ & $1.22 \pm 0.07$ & $0.65 \pm 0.07^{* \dagger}$ \\
\hline \multicolumn{5}{|l|}{ WAT } \\
\hline Ucpl & $1.70 \pm 0.09$ & $3.95 \pm 0.09^{*}$ & $1.74 \pm 0.08$ & $1.95 \pm 0.06^{\dagger}$ \\
\hline Dio2 & $1.46 \pm 0.08$ & $6.10 \pm 0.12^{*}$ & $1.43 \pm 0.05$ & $1.58 \pm 0.09^{\dagger}$ \\
\hline Adrb3 & $1.01 \pm 0.06$ & $3.19 \pm 0.19^{*}$ & $1.21 \pm 0.05$ & $0.93 \pm 0.08^{\dagger}$ \\
\hline$A d r b 2$ & $1.15 \pm 0.05$ & $2.17 \pm 0.10^{*}$ & $1.04 \pm 0.04$ & $0.90 \pm 0.04^{\dagger}$ \\
\hline \multicolumn{5}{|l|}{ Liver } \\
\hline$C d 36$ & $1.24 \pm 0.03$ & $1.35 \pm 0.03$ & $1.60 \pm 0.04^{\dagger}$ & $2.49 \pm 0.06^{* \dagger}$ \\
\hline Fasn & $0.33 \pm 0.02$ & $0.22 \pm 0.02$ & $0.26 \pm 0.03$ & $0.82 \pm 0.03^{* \dagger}$ \\
\hline Ppar $\alpha$ & $1.27 \pm 0.03$ & $1.16 \pm 0.02$ & $1.63 \pm 0.04^{\dagger}$ & $3.01 \pm 0.13^{* \dagger}$ \\
\hline Cpt $1 b$ & $0.94 \pm 0.03$ & $1.05 \pm 0.05$ & $1.61 \pm 0.03^{\dagger}$ & $2.34 \pm 0.05^{* \dagger}$ \\
\hline Pepck & $0.54 \pm 0.05$ & $0.47 \pm 0.03$ & $0.46 \pm 0.07$ & $1.24 \pm 0.06^{* \dagger}$ \\
\hline G6Pase & $0.58 \pm 0.04$ & $0.68 \pm 0.05$ & $0.67 \pm 0.04$ & $0.21 \pm 0.03^{* \dagger}$ \\
\hline
\end{tabular}

Mixed skeletal muscle (gastrocnemius and soleus), WAT and liver were extracted from male mice ( $n=5 /$ genotype) at 5 and 12 months of age to determine the mRNA level of genes by qRT-PCR in duplicate.

Values are expressed as means \pm SEM

${ }^{*} p<0.05$ vs $C c 2^{+/+}$of the same age

${ }^{\dagger} p<0.05$ vs 5 month old mice of the same genotype

Pepck (also known as Pckl) mRNA level in 12 month old mice points to increased gluconeogenesis. However, this does not translate into fasting hyperglycaemia (Table 1). Instead, reduced G6Pase (also known as G6pc) mRNA levels and increased hepatic glycogen content in older fasted (Fig. 5e) and refed mice (Fig. 5f) points to elevated targeting of glucose 6-phosphate to the glycogen synthetic pathways in these mice $[27,29]$.

\section{Discussion}

Previous reports showed that $C \mathrm{C} 2^{-/-}$male mice exhibit hyperphagia that fails to translate into obesity and insulin resistance owing to increased energy expenditure. They also develop increased glucose-induced insulin secretion, at least partly via a mechanism depending on GLP-1 release. This study shows that at $\sim 9$ months of age, male mutant mice develop 

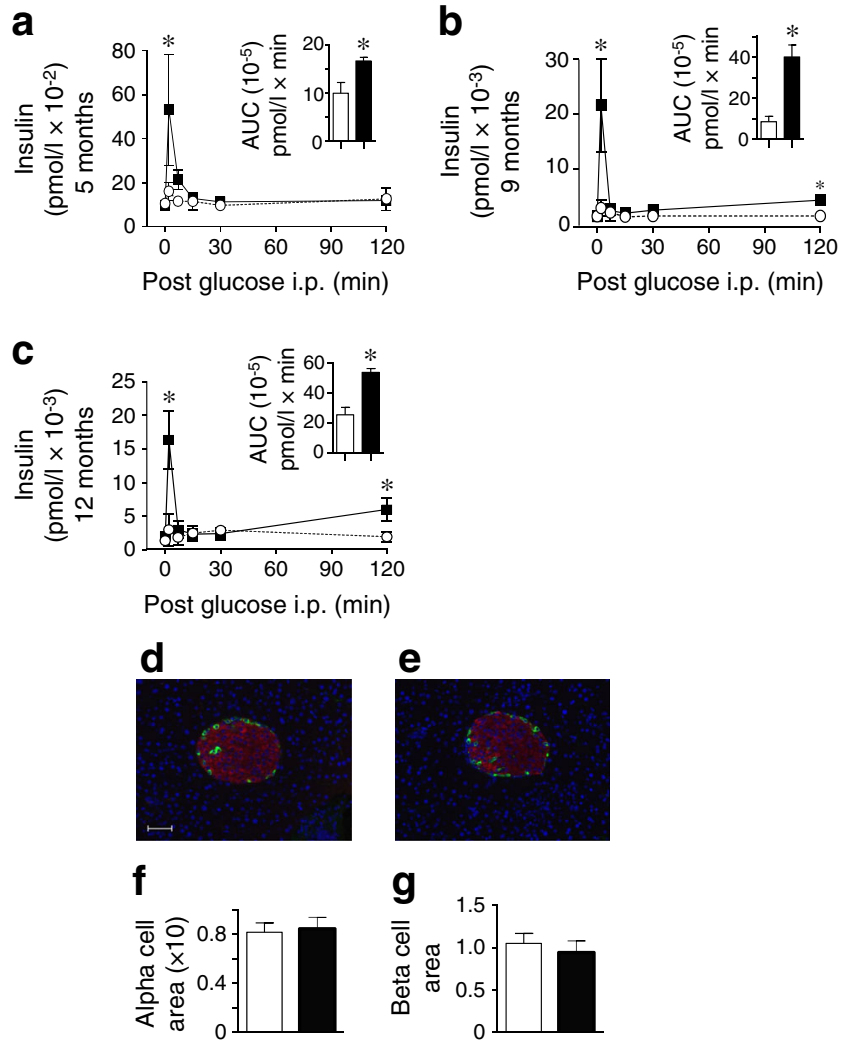

Fig. 4 Insulin secretion and areas of islet cells. (a-c) Glucose-mediated insulin levels were measured at $0-120 \mathrm{~min}$ post i.p. injection of agematched 5-, 9- and 12-month-old mice ( $n \geq 5$ for each genotype and age group). Values are expressed as means \pm SEM. ${ }^{*} p<0.05$ in $\mathrm{Cc}^{-/-}$vs $\mathrm{Cc}^{+/+}$mice. (d-g) Pancreas sections from five of each of 12 month old $C c 2^{+/+}$(d) and $C c 2^{-/-}$(e) mice were fixed and immunostained with antibodies against insulin (red) and glucagon (green) (scale bar, $50 \mu \mathrm{m}$ ). Areas of alpha (f) and beta cells (g) were estimated by morphometric analysis of islets from $C c 2^{+/+}$and $C c 2^{-/-}$mice and calculated relative to pancreas area. Values, expressed as means \pm SEM in arbitrary units, are presented in the bar graphs below. Black squares and bars, $C c 2^{-/-}$; white circles and bars, $C c 2^{+/+}$

hyperphagia-driven insulin resistance. They also manifest a decline in energy expenditure and physical activity. As summarised in Fig. 6, this energy imbalance occurs together with impaired hepatic insulin clearance that sustains chronic hyperinsulinaemia, at least in part by failing to offset the persistent increase in insulin secretion.

While hyperphagia occurs in both $C c 2^{-/-}$male and female mice, the age at which its resulting insulin resistance emerges differs between groups, with female mice developing metabolic derangement at 2 months [6] and male mice at $~ 9$ months of age, as the current studies demonstrate. The delay in insulin resistance in male mice is attributed to the counter-regulatory effect of their early hypermetabolic state that offsets hyperphagia and maintains insulin sensitivity at a young age [15]. This includes complete fatty acid $\beta$-oxidation in skeletal muscle, a main site of energy expenditure, and increased brown adipogenesis and sympathetic tone to adipose tissue [15] (Fig. 6). In older mice, however, fatty acid $\beta$-oxidation in skeletal muscle
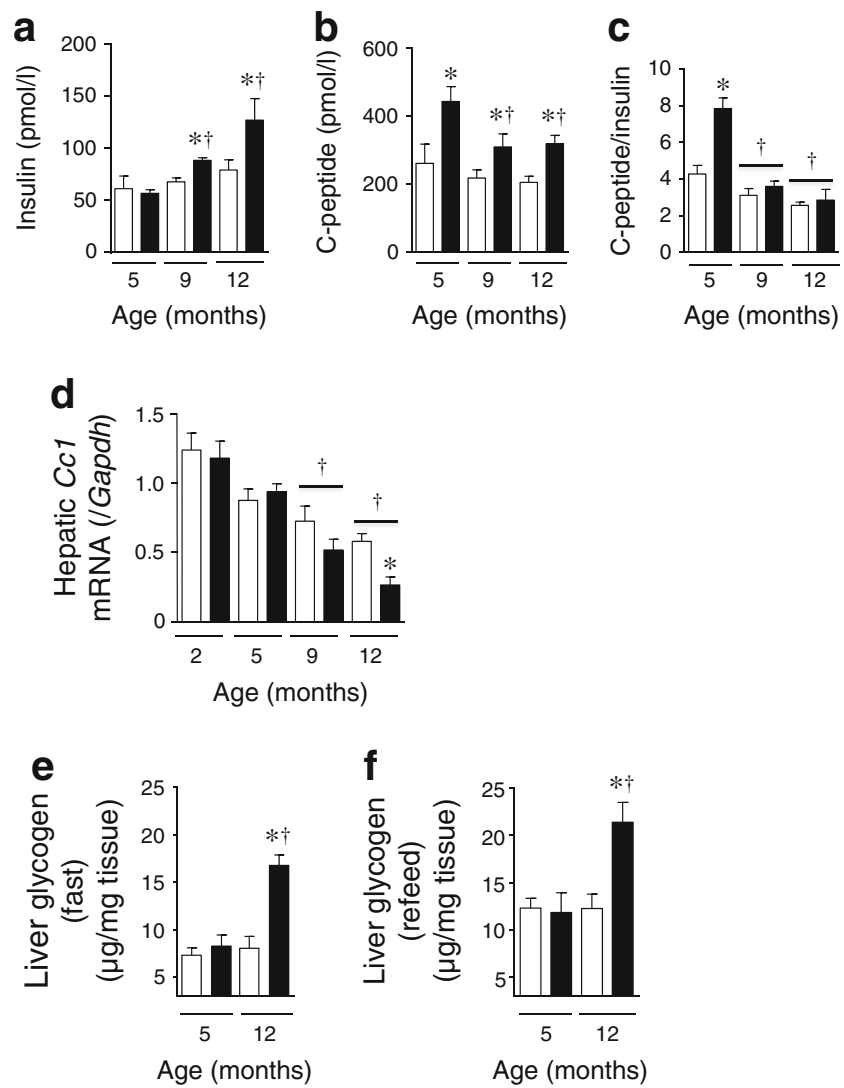

Fig. 5 Hepatic insulin clearance and glycogen content. (a-c) $C c 2^{-/-}$and $\mathrm{Cc}^{+/+}$mice were fasted overnight until 11:00 $\mathrm{h}$ on the next day to determine steady-state plasma insulin (a) and C-peptide (b) levels in triplicate and calculate the $\mathrm{C}$-peptide/insulin molar ratio (c) as a surrogate measure of insulin clearance ( $n \geq 6$ mice for each genotype and age group). (d) Ccl mRNA content was analysed in triplicate in liver tissues from mice aged $2-12$ months ( $n \geq 5$ for each genotype and age group). (e, f) Hepatic glycogen content was assayed in triplicate in overnight-fasted (e) and refed (f) mice ( $n \geq 5$ mice for each subgroup). Values are expressed as means \pm SEM. White, $C c 2^{+/+}$; black, $C c 2^{-/-} \cdot{ }^{*} p<0.05 \mathrm{vs} C c 2^{+/+}$of the same age group. ${ }^{\dagger} p<0.05$ vs mice at the earliest age examined

decreases at $\sim 9$ months of age, leading to lipid accumulation and lipotoxicity. As in other murine models of altered metabolism [30, 31], this could contribute to insulin resistance in skeletal muscle, as manifested by glucose intolerance and underlined by reduced insulin-stimulated Akt phosphorylation, a key mediator of glucose uptake [32]. Glucose uptake in skeletal muscle becomes limited in parallel to an agedependent reduction in metabolically active lean mass and an increase in visceral obesity [33]. Consistent with this, lean mass decreased reciprocally to the increase in fat mass in older $\mathrm{Cc}^{-/-}$male mice. Given that CEACAM2 is not expressed in skeletal muscle [9], it is likely that the progressive decrease in energy expenditure in older mutants is caused by altered central regulation of glucose uptake, resulting from loss of CEACAM2 in the VMH [6], a main central regulator of glucose uptake in skeletal muscle that acts via the sympathetic nervous system [24]. 


\section{a Male $C c 2^{-/}$(5 months)}

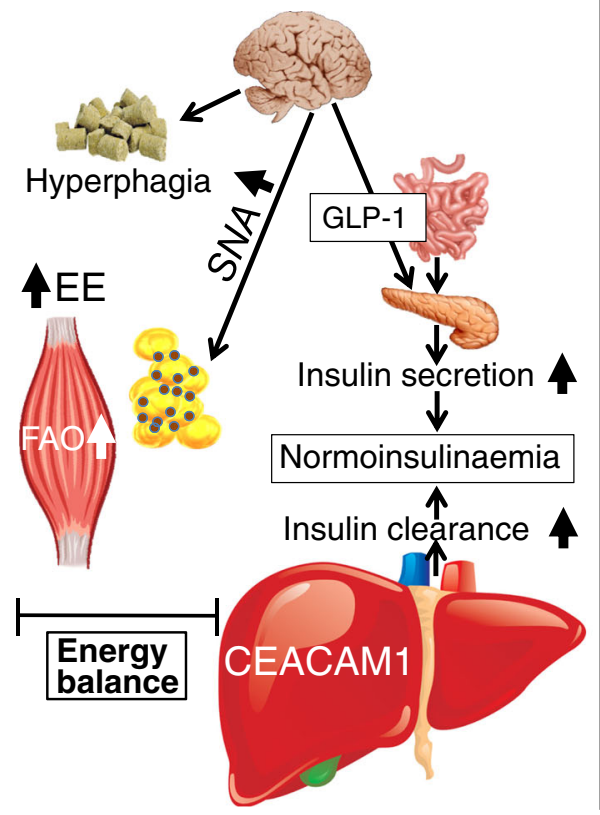

Fig. 6 Metabolic regulation in 5 month old (a) and 9 month old (b) $C c 2^{-1-}$ male mice. Despite hyperphagia caused by $C c 2$ null deletion, younger $C c 2^{-1-}$ mice (a) manifest a hypermetabolic state that includes an increase in energy expenditure and fatty acid $\beta$-oxidation in skeletal muscle, increased brown adipogenesis of the white adipose depot (brown dots) and elevated sympathetic nervous system activation. Null deletion of $C c 2$ leads to an increase in insulin secretion mediated by GLP-1 release. This induces hepatic CEACAM1 expression to promote insulin clearance and maintain insulin at a physiological level to sustain insulin sensitivity. A metabolic switch occurs at an older age starting at $\sim 9$ months of age (b). This includes reduced brown adipogenesis and sympathetic tone in adipose tissue, which leads to visceral obesity and increased

Moreover, young male $C \mathrm{c} 2^{-/-}$mice exhibit increased sympathetic tone to WAT, consistent with increased brown adipogenesis in this depot and higher energy dissipation [15]. In line with this, our study showed that they have increased expression of the surrogate markers of brown adipogenesis (Ucp1 and Dio2) [25] and activated sympathetic tone (Ucp1, Adrb2 and Adrb3) [23] in their WAT. In contrast, these markers are significantly reduced in older mutants. Considering that CEACAM2 is not detected physiologically in adipose tissue [9], reduced energy dissipation and sympathetic tone in adipose tissues of aged $\mathrm{Cc}^{-/-}$male mice is probably mediated by its deletion from brain nuclei participating in the neuroendocrine regulation of energy balance, such as VMH $[34,35]$.

Insulin resistance is heralded by chronic hyperinsulinaemia. $C c 2$ deletion causes an increase in insulin secretion and an amplified acute excursion of insulin in response to glucose across all age groups in male mutant mice. Elevated insulin secretion is in part mediated by increased release of GLP-1 [12], an incretin that potentiates glucose-stimulated insulin secretion from pancreatic beta cells [36-38]. This is supported by persistently b Male $C c 2^{--}$(9 months)

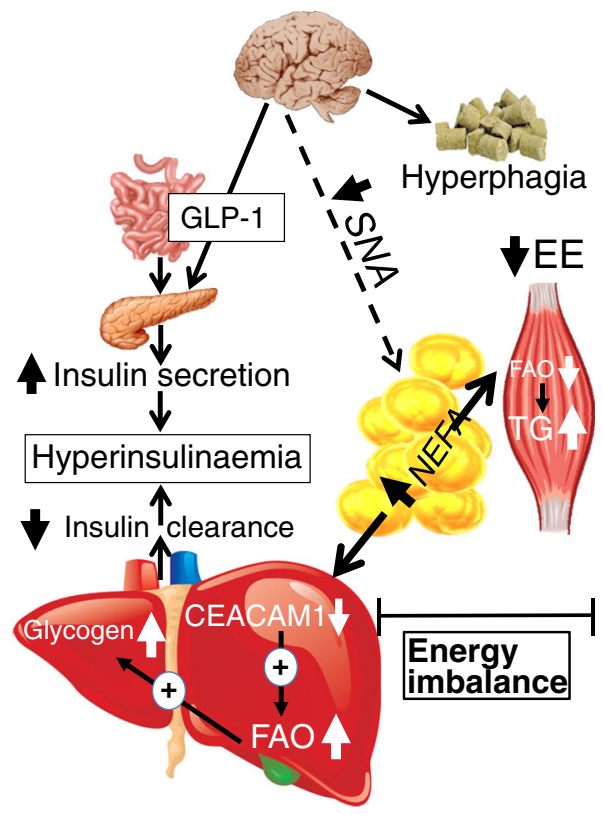

lipolysis and NEFA release. Excessive NEFA transport reduces fatty acid $\beta$-oxidation in skeletal muscle and consequently, increases triacylglycerol accumulation and insulin resistance in muscle. Additionally, NEFA transport to the liver reduces hepatic CEACAM1 levels, an event that provides a positive feedback mechanism on fatty acid $\beta$-oxidation. The resulting increase in citrate production and its redistribution to the cytoplasm lead to a rise in glucose 6-phosphate and its re-routing to the glycogen repletion pathways. Moreover, the decrease in CEACAM1 mediates a decrease in insulin clearance, which fails to counter the increase in insulin secretion, leading to chronic hyperinsulinaemia and sustained systemic insulin resistance. EE, energy expenditure; FAO, fatty acid $\beta$-oxidation; SNA, sympathetic nervous system activation

higher plasma GLP-1 levels and an intact beta cell area at 12 months of age.

Despite the increase in insulin secretion caused by $\mathrm{Cc} 2$ deletion at an early age, chronic hyperinsulinaemia does not develop in mutant males until $\sim 9$ months. At an earlier age, elevated insulin secretion is countered by an increase in hepatic insulin clearance as shown by the approximately twofold higher steady-state plasma C-peptide/insulin molar ratio in the young mutants. This is probably mediated by the induction of hepatic CEACAM1 production by insulin, which increases the transcription of the $C c 1$ promoter [26] to elevate mRNA and protein levels of CEACAM1 [27]. At 9 months of age, however, insulin clearance begins to decline substantially, probably mediated by a progressive decrease in hepatic CEACAM1 expression. Whereas this remarkable decrease in $C c 1$ mRNA could stem from a compromised ability of insulin to induce $C c 1$ transcription under conditions of hyperphagia-driven insulin resistance, it could also result from the activation of PPAR $\alpha$ by fatty acids that are mobilised from visceral adipose tissue $[27,39]$. The decrease in hepatic CEACAM1 level provides a positive feedback effect on fatty 
acid $\beta$-oxidation [27], and as such prevents lipid accumulation in aged $\mathrm{Cc}^{-/-}$livers. Additionally, an increase in fatty acid $\beta$-oxidation produces acetyl-CoA that inhibits pyruvate dehydrogenase to prevent glycolysis and reroute pyruvate to gluconeogenesis (supported by increased Pepck mRNA levels). This also causes cytoplasmic citrate to inhibit 6-phosphofructo-1-kinase, increasing glucose 6-phosphate production. With reduced glucose-6-phospatase levels, glucose 6-phosphate is rerouted to the glycogen synthetic pathways $[27,29]$. This is consistent with a role for CEACAM1 in hepatic gluconeogenesis via regulating $\beta$-oxidation of lipolysis-derived fatty acids [40], thus limiting fasting hyperglycaemia that also, in part, results from increased insulin secretion.

Whereas age-related hyperinsulinaemia, insulin resistance and visceral obesity [41-43] are commonly associated with reduced glucose uptake in skeletal muscle in parallel with a reduction in metabolically active lean mass [33], decreased fenestration in the liver sinusoidal endothelium and other structural changes that reduce insulin uptake by hepatocytes, and hence reduce insulin clearance, have recently emerged as mechanisms underlying age-related hyperinsulinaemia and insulin resistance [44]. The current study provides an alternative mechanism of hyperinsulinaemia in older $C_{c} 2^{-/-}$male mutant mice, implicating a progressive reduction in hepatic CEACAM1-mediated insulin clearance that fails to counter persistent GLP-1-mediated release of insulin in older mutant mice. This points to compromised hepatic insulin clearance as a major contributor to the chronic hyperinsulinaemic state that develops in older $C c 2^{-/-}$male mice, in agreement with several clinical studies showing reduction in insulin clearance as a critical player in the pathogenesis of metabolic abnormalities associated with obesity, glucose intolerance and insulin resistance [45-47].

More studies are needed to delineate the role of the decline in hepatic CEACAM1 levels with age, but the current study is the first to describe how altered hepatic insulin clearance might contribute to age-related insulin resistance (Fig. 6). More specifically, the data suggest that loss of CEACAM2 from critical brain centres involved in the regulation of food intake causes hyperphagia, followed by insulin resistance (including lipolysis). This lowers hepatic CEACAM1 level and phosphorylation to impair hepatic insulin clearance and cause an unmatched increase in insulin secretion, leading to chronic hyperinsulinaemia and sustained insulin resistance with age.

Acknowledgements The authors thank M. Kopfman at the Najjar Laboratory for her technical assistance with the generation and maintenance of mice, and in carrying out routine DNA and RNA analyses.

Data availability Data are available on request from the authors.
Funding This work was supported by grants from the National Institutes of Health, R01-DK-054254, R01-DK-083850 and R01-HL112248 to SMN and R01 DK67536 and R01 DK103215 to RNK, and from the Middle-East Diabetes Research Center, to SSG.

Duality of interest The authors declare that there is no duality of interest associated with this manuscript.

Contribution statement SSG researched data, designed experiments, wrote a first draft and revised the manuscript. HTM, AMD, JH and GH researched data and revised the manuscript critically. GH was involved in data analysis and discussions. He also critically read the manuscript. RNK supervised the experiments pertaining to pancreatic cell areas, analysed data, and reviewed critically the manuscript. SMN was responsible for study design, conceptualisation, data analysis and results interpretation, and reviewing the manuscript. SMN had full access to all the data of the study and takes responsibility for the integrity and accuracy of data analysis and the decision to submit and publish the manuscript. All authors have given final approval of this version to be published.

\section{References}

1. Kuespert K, Pils S, Hauck CR (2006) CEACAMs: their role in physiology and pathophysiology. Curr Opin Cell Biol 18:565-571

2. Robitaille J, Izzi L, Daniels E, Zelus B, Holmes KV, Beauchemin N (1999) Comparison of expression patterns and cell adhesion properties of the mouse biliary glycoproteins Bbgp1 and Bbgp2. ?Eur J Biochem 264:534-544

3. Najjar SM (2002) Regulation of insulin action by CEACAM1. Trends Endocrinol Metab 13:240-245

4. Poy MN, Yang Y, Rezaei K et al (2002) CEACAM1 regulates insulin clearance in liver. Nat Genet 30:270-276

5. DeAngelis AM, Heinrich G, Dai T et al (2008) Carcinoembryonic antigen-related cell adhesion molecule 1: a link between insulin and lipid metabolism. Diabetes 57:2296-2303

6. Heinrich G, Ghosh S, Deangelis AM et al (2010) Carcinoembryonic antigen-related cell adhesion molecule 2 controls energy balance and peripheral insulin action in mice. Gastroenterology 139:644-652

7. Salaheldeen E, Kurio H, Howida A, Iida H (2012) Molecular cloning and localization of a CEACAM2 isoform, CEACAM2-L, expressed in spermatids in mouse testis. Mol Reprod Dev 79: $843-852$

8. Alshahrani MM, Yang E, Yip J et al (2014) CEACAM2 negatively regulates hemi (ITAM-bearing) GPVI and CLEC-2 pathways and thrombus growth in vitro and in vivo. Blood 124:2431-2441

9. Han E, Phan D, Lo P et al (2001) Differences in tissue-specific and embryonic expression of mouse Ceacam1 and Ceacam2 genes. Biochem J 355:417-423

10. Nedellec P, Dveksler GS, Daniels E et al (1994) Bgp2, a new member of the carcinoembryonic antigen-related gene family, encodes an alternative receptor for mouse hepatitis viruses. J Virol 68:45254537

11. Zebhauser R, Kammerer R, Eisenried A, McLellan A, Moore T, Zimmermann W (2005) Identification of a novel group of evolutionarily conserved members within the rapidly diverging murine Cea family. Genomics 86:566-580

12. Ghanem SS, Heinrich G, Lester SG et al (2016) Increased glucoseinduced secretion of glucagon-like peptide-1 in mice lacking the carcinoembryonic antigen-related cell adhesion molecule 2 (CEACAM2). J Biol Chem 291:980-988

13. Morton GJ, Cummings DE, Baskin DG, Barsh GS, Schwartz MW (2006) Central nervous system control of food intake and body weight. Nature 443:289-295 
14. Cota D, Barrera JG, Seeley RJ (2006) Leptin in energy balance and reward: two faces of the same coin? Neuron 51:678-680

15. Patel PR, Ramakrishnan SK, Kaw MK et al (2012) Increased metabolic rate and insulin sensitivity in male mice lacking the carcinoembryonic antigen-related cell adhesion molecule 2. Diabetologia $55: 763-772$

16. Salaheldeen E, Howida A, Wakayama T, Iida H (2014) CEACAM2-L on spermatids interacts with poliovirus receptor on Sertoli cells in mouse seminiferous epithelium. J Histochem Cytochem 62:632-644

17. Bingham NC, Anderson KK, Reuter AL, Stallings NR, Parker KL (2008) Selective loss of leptin receptors in the ventromedial hypothalamic nucleus results in increased adiposity and a metabolic syndrome. Endocrinology 149:2138-2148

18. Al-Share QY, DeAngelis AM, Lester SG et al (2015) Forced hepatic overexpression of CEACAM1 curtails diet-induced insulin resistance. Diabetes 64:2780-2790

19. Park SY, Cho YR, Kim HJ et al (2006) Mechanism of glucose intolerance in mice with dominant negative mutation of CEACAM1. Am J Physiol Endocrinol Metab 291:E517-E524

20. Morioka T, Asilmaz E, Hu J et al (2007) Disruption of leptin receptor expression in the pancreas directly affects beta cell growth and function in mice. J Clin Invest 117:2860-2868

21. Michael MD, Kulkarni RN, Postic C et al (2000) Loss of insulin signaling in hepatocytes leads to severe insulin resistance and progressive hepatic dysfunction. Mol Cell 6:87-97

22. Kulkarni RN, Almind K, Goren HJ et al (2003) Impact of genetic background on development of hyperinsulinemia and diabetes in insulin receptor/insulin receptor substrate-1 double heterozygous mice. Diabetes 52:1528-1534

23. Onai T, Kilroy G, York DA, Bray GA (1995) Regulation of beta 3adrenergic receptor mRNA by sympathetic nerves and glucocorticoids in BAT of Zucker obese rats. Am J Phys Regul Integr Comp Phys 269:R519-R526

24. Shiuchi T, Haque MS, Okamoto S et al (2009) Hypothalamic orexin stimulates feeding-associated glucose utilization in skeletal muscle via sympathetic nervous system. Cell Metab 10:466-480

25. de Jesus LA, Carvalho SD, Ribeiro MO et al (2001) The type 2 iodothyronine deiodinase is essential for adaptive thermogenesis in brown adipose tissue. J Clin Invest 108:1379-1385

26. Najjar S, Boisclair Y, Nabih Z et al (1996) Cloning and characterization of a functional promoter of the rat pp120 gene, encoding a substrate of the insulin receptor tyrosine kinase. J Biol Chem 271: 8809-8817

27. Ramakrishnan SK, Khuder SS, Al-Share QY et al (2016) PPAR $\alpha$ (peroxisome proliferator-activated receptor alpha) activation reduces hepatic CEACAM1 protein expression to regulate fatty acid oxidation during fasting-refeeding transition. J Biol Chem 291: 8121-8129

28. DeFronzo RA (1988) Lilly lecture 1987. The triumvirate: beta-cell, muscle, liver. A collusion responsible for NIDDM. Diabetes 37: 667-687

29. Hue L, Taegtmeyer H (2009) The Randle cycle revisited: a new head for an old hat. Am J Physiol Endocrinol Metab 297:E578E591
30. Koves TR, Ussher JR, Noland RC et al (2008) Mitochondrial overload and incomplete fatty acid oxidation contribute to skeletal muscle insulin resistance. Cell Metab 7:45-56

31. Wang H, Knaub LA, Jensen DR et al (2009) Skeletal musclespecific deletion of lipoprotein lipase enhances insulin signaling in skeletal muscle but causes insulin resistance in liver and other tissues. Diabetes 58:116-124

32. Summers SA, Yin VP, Whiteman EL et al (1999) Signaling pathways mediating insulin-stimulated glucose transport. Ann N Y Acad Sci 892:169-186

33. Yalamanchi SV, Stewart KJ, Ji N et al (2016) The relationship of fasting hyperglycemia to changes in fat and muscle mass after exercise training in type 2 diabetes. Diabetes Res Clin Pract 122:154 161

34. Saito M, Minokoshi Y, Shimazu T (1985) Brown adipose tissue after ventromedial hypothalamic lesions in rats. Am J Physiol Endocrinol Metab 248:E20-E25

35. King BM (2006) The rise, fall, and resurrection of the ventromedial hypothalamus in the regulation of feeding behavior and body weight. Physiol Behav 87:221-244

36. D’Alessio DA, Kahn SE, Leusner CR, Ensinck JW (1994) Glucagon-like peptide 1 enhances glucose tolerance both by stimulation of insulin release and by increasing insulin-independent glucose disposal. J Clin Invest 93:2263-2266

37. Reimann F, Gribble FM (2002) Glucose-sensing in glucagon-like peptide-1-secreting cells. Diabetes 51:2757-2763

38. Habib AM, Richards P, Rogers GJ, Reimann F, Gribble FM (2013) Co-localisation and secretion of glucagon-like peptide 1 and peptide YY from primary cultured human L cells. Diabetologia 56: 1413-1416

39. Ramakrishnan SK, Russo L, Ghanem SS et al (2016) Fenofibrate decreases insulin clearance and insulin secretion to maintain insulin sensitivity. J Biol Chem 291:23915-23924

40. Titchenell PM, Quinn WJ, Lu M et al (2016) Direct hepatocyte insulin signaling is required for lipogenesis but is dispensable for the suppression of glucose production. Cell Metab 23:1154-1166

41. Fink RI, Kolterman OG, Griffin J, Olefsky JM (1983) Mechanisms of insulin resistance in aging. J Clin Invest 71:1523-1535

42. Short KR, Vittone JL, Bigelow ML et al (2003) Impact of aerobic exercise training on age-related changes in insulin sensitivity and muscle oxidative capacity. Diabetes 52:1888-1896

43. Oya J, Nakagami T, Yamamoto Y et al (2014) Effects of age on insulin resistance and secretion in subjects without diabetes. Intern Med 53:941-947

44. Mohamad M, Mitchell SJ, Wu LE et al (2016) Ultrastructure of the liver microcirculation influences hepatic and systemic insulin activity and provides a mechanism for age-related insulin resistance. Aging Cell 15:706-715

45. Valera Mora ME, Scarfone A, Calvani M, Greco AV, Mingrone G (2003) Insulin clearance in obesity. J Am Coll Nutr 22:487-493

46. Lee CC, Haffner SM, Wagenknecht LE et al (2013) Insulin clearance and the incidence of type 2 diabetes in Hispanics and African Americans: the IRAS family study. Diabetes Care 36:901-907

47. Marini MA, Frontoni S, Succurro E et al (2014) Differences in insulin clearance between metabolically healthy and unhealthy obese subjects. Acta Diabetol 51:257-261 